\title{
La formacion practica en enfermería un estudio cualitativo en los internos que cursan sus prácticas clínicas.
}

The practical training in nursing a qualitative study in the inmates studying clinical practices.

Lic. Joicy Anabel Franco Coffré. Mg. ${ }^{1}$, Lic. Ruth Jackeline Oviedo Rodriguez. MSc. ${ }^{2}$, Lic. Ketty Aracely Piedra Chavez Mg. ${ }^{3}$ \& Lic. Aleymis Torres Chils MSc. ${ }^{4}$

\begin{abstract}
.
DOI: https://doi.org/10.33262/cienciadigital.v2i1.4

The purpose of this study was to analyze the perceptions of nursing students about their clinical practice. The research focus was the phenomenological qualitative based on the model of Patricia Benner. Through a semi-structured interview, information was gathered from seven nursing students who were in their clinical practice year. Negative feelings were identified as anxiety, insecurity and impotence due to the lack of skills acquired during undergraduate. Positive feelings of help to the patient, acquisition of new skills, overcoming fears, confidence, autonomy and safety as the days passed. The students in their entirety asserted that the learning generator was the service nurse while what impeded was the absence of the university teacher. The quantity and quality of the practical component in the undergraduate program was questionable. It is recommended as a strategy to include the graduate in nursing care as an ally in the learning process
\end{abstract}

Keywords: Boarding School, Residence, Nursing, Education in Nursing, Hospital, Nursing Student.

\footnotetext{
${ }^{1}$ Universidad de Guayaquil, Carrera de Enfermería, Guayaquil - Ecuador, joicy.francoc@ug.edu.ec ${ }^{2}$ Universidad de Guayaquil, Carrera de Enfermería, Guayaquil - Ecuador, ruth.oviedor@ug.edu.ec ${ }^{3}$ Universidad de Guayaquil, Carrera de Enfermería, Guayaquil - Ecuador, piedrack@ug.edu.ec ${ }^{4}$ Universidad de Guayaquil, Carrera de Enfermería, Guayaquil - Ecuador, aleymis.torresc@ug.edu.ec
} 


\section{Resumen}

La finalidad de este estudio fue analizar las percepciones de los estudiantes de enfermería sobre su práctica clínica. El enfoque de investigación fue el cualitativo fenomenológico basado en el modelo de Patricia Benner. Mediante entrevista semiestructurada se recolectó la información de siete estudiantes de enfermería que cursaban su año de práctica clínica. Se identificaron sensaciones negativas como ansiedad, inseguridad e impotencia por la falta de destrezas adquiridas durante el pregrado. Sensaciones positivas de sentido de ayuda al paciente, adquisición de nuevas habilidades, superación de temores, confianza, autonomía y seguridad conforme trascurrían los días. Los estudiantes en su totalidad aseveraron que el factor generador de aprendizaje fue la enfermera de servicio mientras que lo que obstaculizaba fue la ausencia del docente universitario. La cantidad y calidad del componente práctico en el pregrado fue cuestionable. Se recomienda como estrategia incluir la licenciada en enfermería asistencial como aliada en el proceso de aprendizaje.

Palabras Claves: Internado, Residencia, Enfermería, Educación en Enfermería, Hospital, Estudiante de Enfermería

\section{Introducción.}

El aprendizaje según la real academia de la lengua "es la acción y efecto de aprender algún arte, oficio, u otra cosa", y desde el punto de vista psicológico, se define como "la adquisición por la práctica, de una conducta duradera" Briceño, V. (2011)., interesantes visiones que confirman que la teoría y la práctica van de la mano en el proceso de aprendizaje como tal. El conocimiento adquirido está en la acción y se revela a través de la ejecución espontánea y hábil del dueño de dicho conocimiento. No hay nada más práctico que una buena teoría, ésta debería de forma insoslayable, verse vinculada a la práctica pero cuando estas se divorcian o fracturan, con total certeza existe falta de aprendizaje.

Complementando lo anterior, la teoría dirige a la práctica de un modo ordenado y sistemático, la práctica, sirve para comprender la teoría y confirmarla pero no siempre es así. La experiencia vivenciado por los estudiantes al aplicar la teoría, en ocasiones, podrían llevarlos a reelaborar los conocimientos aprendidos, teóricos puros, por nuevas o diferentes consecuencias obtenidas, y que en ocasiones anulan ese saber áulico.

Una de las principales frustraciones que podría tener un docente, es que sus alumnos no hayan aprendido. Existen cuatro tipos de conocimientos de los estudiantes: el conocimiento olvidado, el conocimiento inerte, el conocimiento ritual y el conocimiento ingenuo, Pogre, P. (2017). Un conocimiento olvidado es aquel que ha desaparecido de la mente del estudiante por completo. El conocimiento inerte es aquel que cuando se toma algún examen el estudiante lo recuerda bien, pero es incapaz de usarlo en situaciones de la vida real. El conocimiento 
ingenuo, es aquel que se capta superficialmente en la mayor parte de la teoría, pero se suele tener ideas ingenuas acerca del mismo. Finalmente el conocimiento ritual es cuando los alumnos no entienden lo que se les enseña o al menos no por completo, y tratan de compensar aquello con rituales que funcionan bien en el mundo artificial del salón de clases.

Ya sea que el estudiante olvide lo que se le enseñó, o que simplemente realice rituales para tratar de compensar sus vacíos, nos chocamos con la idea de que no se forman profesionales competentes, Altmann, T.(2007). El concepto de una persona competente, radica básicamente en que es aquella que además de poseer conocimientos y destrezas técnicas posee también capacidades prácticas o psicosociales requeridas ante las situaciones que se enfrente el día a día. Las competencias y capacidades no se enseñan ni aprende, sino que se construyen y desarrollan en la resolución de problemas en la práctica diaria.

En el ámbito de la salud y más aun de la enfermería, la no combinación de la teoría y la práctica es inexcusable. La enfermería es una disciplina basada en la práctica, un estudiante con bases teóricas es de sustancial importancia, pero las habilidades adquiridas durante los años de pregrado, pensamiento crítico y reflexivo prometen un mejor desenvolvimiento durante la vida profesional y mayor calidad en la prestación de los cuidados Morán, L. (2016).

Dentro de los modelos de enfermería que existen, Benner, P. (1982)., conocida teórica de enfermería, pero en la actualidad, denominada más como filósofa entre sus constructos se encontraba el precepto de que la enfermera a medida que camina por su vida profesional, va desarrollando experticia. Este nivel de experiencia se desarrolla en los siguiente niveles: 1. Principiante, 2. Principiante avanzado, 3. Competente, 4. Proficiente 5. Experto. El principiante es aquel que se le debe decir lo que tiene que hacer y lo hará. El principiante avanzado tiene un rendimiento aceptable y adquiere principios basado en sus experiencias reales. La enfermera competente tiende a ser la que ya tiene una experiencia laboral en un mismo ambiente de trabajo por al menos 2 a 3 años. En el nivel proficiente la experiencia enseña a la enfermera cuáles son los eventos típicos dada la situación y cómo modificar los planes en respuesta a estos eventos. La enfermera experta en cambio, no depende de reglas ni directrices, basa su accionar en las experiencias que le han dado resultado siempre, con comprensión intuitiva y alto grado de competencia Cárdenas, B.(2014).

La Filosofía de Patricia Benner muestra el progreso que atraviesa la enfermera recién graduada hasta la especialista en un área determinada. Esto conlleva cambios de conducta, adquisición de habilidades y destrezas lo cual repercute en desempeños de mejor calidad Carrillo A.(2017).

A efectos del presente artículo en el que se describe las percepciones de estudiantes de enfermería en su práctica clínica, se vuelve pertinente la filosofía de Patricia Benner ya que 
el estudiante en cuestión pasa por los primeros niveles de desarrollo profesional, y así la visión y análisis que se den a sus respuestas podrían ser factores de encasillamiento a su nivel de experticia.

El objetivo de esta investigación es ilustrar la percepción, reflexión y relación que tiene el conocimiento teórico aplicado por los internos de enfermería en las prácticas pre profesionales desde la visión del modelo teórico de Patricia Benner.

\section{Métodos.}

Investigación de enfoque cualitativo, y de tipo fenomenológico ya que pretende comprender y profundizar los fenómenos, explorándolos desde la perspectiva de los participantes en un ambiente natural y en relación con el contexto Onwuegbuzie, A. (2013). Los métodos cualitativos son humanistas, cuando uno utiliza el método cuantitativo reducimos a los actos y palabras de la gente a ecuaciones estadísticas, se pierde de vista el lado humano. Por medio de la investigación cualitativa llegamos a conocer las personas en su individualidad y a experimentar lo que ellos sienten en sus luchas cotidianas Cadenas, D.(2016). El escenario de investigación fue la carrera de enfermería de la Universidad de Guayaquil-Ecuador y los sujetos de investigación fueron los internos de enfermería que cursaban sus prácticas clínicas.

La técnica de captación de los participantes fue selección por conveniencia (estudiantes que cursaban el último año de la carrera de enfermería, es decir realizando su internado rotativo). Durante el periodo de 3 meses fueron entrevistados 7 sujetos de estudio, a los cuales se aplicó entrevista semiestructurada de forma individual para que pudiesen sentirse en plena confianza de responder sin ser influidos por nadie en sus respuestas, como podía haber sucedido si se utilizaba como técnica el grupo focal. El tiempo de duración fue de 20 minutos, y los participantes decidieron día, hora y lugar que conforme al rigor ético, fueron lugares reservados, silenciosos y que no representaran interrupciones y con previa autorización de la directora de la carrera y firma de consentimiento informado por los participantes.

El instrumento para la recolección de los datos fue la entrevista semiestructurada puesto que presenta un grado mayor de flexibilidad que las estructuradas, debido a que parten de preguntas planeadas, que pueden ajustarse a los entrevistados Carrera, H. (2014). Su ventaja es la posibilidad de adaptarse a los sujetos con enormes posibilidades para motivar al interlocutor, aclarar términos, identificar ambigüedades y reducir formalismos.

Las preguntas fueron abiertas y se encontraban separadas por temáticas: Sobre las sensaciones positivas, sensaciones negativas, sobre la aplicación de la teoría y la práctica y sobre la universidad. Tales preguntas correspondieron a las siguientes: ¿Cuáles han sido las vivencias agradables y desagradables experimentadas durante su práctica clínica?, ¿qué 
experiencias de la práctica causaron preocupación en usted? ¿Le ha costado aplicar lo aprendido teóricamente en la práctica? Si la respuesta es sí, cual fue esta experiencia. ¿Cuáles fueron los obstáculos para el aprendizaje durante la práctica clínica? ¿Cuáles fueron los facilitadores de aprendizaje durante la práctica clínica? ¿Qué debilidad crees que tienes como interno de enfermería? ¿Cómo cree usted que debería mejora la preparación en la universidad concerniente a la práctica clínica?

Las preguntas de la entrevista fueron validadas por dos expertas en investigación cualitativa con formación de $\mathrm{PhD}$. quienes hicieron sugerencias con relación a las preguntas y contenido teórico, estas se pusieron a consideración. Se realizó prueba piloto con un grupo de 5 estudiantes donde se pudo evidenciar la facilidad de entendimiento de las preguntas del instrumento, y así se pudo ya ejecutar las entrevistas.

El análisis de los datos fue fundamento en el análisis temático de contenido. Siendo agrupados en el sistema de análisis categorial. Una vez fueron transcritas las entrevistas de forma textual (datos brutos) se les asigno un código a cada participante: P1, P2, P3, de acuerdo al orden cronológico, para preservar la identidad de cada uno de ellos. Posteriormente se aplicó con un rotulador de colores cada frase interesante y se codifico dicha frase. Seguidamente se dio el proceso de transformación de los códigos hacia categorías mediante el análisis categorial de contenido de cada participante relacionado con sus respuestas de la entrevista.

Finalmente, como resultado del análisis de contenido, surgen cuatro categorías empíricas denominadas: la brecha entre teoría y práctica, Sensación de incompetencia e inseguridad en la realización de procedimientos, ansiedad inicial por las relaciones interpersonales y profesionales, rol profesional versus rol de ayudante del auxiliar de enfermería, insatisfacción con la cantidad de práctica en los años universitarios. Estas serán discutidas a continuación.

\section{Resultados y discusión.}

Antes de desarrollar los resultados y discusión, es apropiado presentar la caracterización de los participantes. Fueron 7 participantes en total, seis mujeres y un hombre. Dos de ellos realizaron su práctica clínica en hospitales de segundo nivel de atención y 6 de ellos en hospitales de tercer nivel. Sus edades oscilaban entre 23 y 24 años de edad, estado civil soltero, seis de ellos nacidos en la ciudad de Guayaquil y uno proveniente de otra ciudad del Ecuador.

Por medio del proceso de análisis de contenido, emergieron cinco categorías empíricas denominadas: la brecha entre teoría y práctica, Sensación de incompetencia e inseguridad en la realización de procedimientos, ansiedad inicial por las relaciones interpersonales y 
profesionales, rol profesional versus rol de ayudante del auxiliar de enfermería, insatisfacción con la cantidad de práctica en los años universitarios.

\section{La brecha entre teoría y práctica.}

Entre los desafíos más grandes que posee un profesor, se encuentra que sus estudiantes sean competentes y sepan aplicar la teoría áulica en la práctica profesional. En las entrevistas realizadas se pudo detectar quienes manifestaban tener dificultad con la aplicación del conocimiento, no por no haberlo aprendido bien, sino por los contextos del entorno en el que se encontraban. Una estudiante aseveró: Nos dicen en la universidad, que se yo, hay que administrar medicación con tal tipo de dilución pero en el hospital si no hay uno tiene que improvisar, es verdad, que hay que tener más cuidado en el momento de la administración sí, pero también uno debe tener en cuenta que hay que ser improvisado para hacer muchas cosas porque no hay. La asepsia, administración de medicamentos, al mismo tiempo el cuidado a veces uno no tiene como monitorizar al paciente, entonces solo se lo controla con un saturador o con monitores manuales. (P1)

La falta de insumos, materiales de trabajo y demás, son situaciones comunes en los hospitales públicos pertenecientes a los países en vías de desarrollo. Se hace lo que se puede con lo que se tiene, y el interno de enfermería contrasta lo aprendido en la literatura y demostrado por el profesor universitario. Se suele compensar esa falta de recursos con la omnipotencia de las personas que suple las carencias estructurales del sistema con la voluntad de trabajo, Martínez, R. (2016). Al llegar a la práctica clínica y encontrar que todo lo dicho teóricamente queda en una irrealidad, el estudiante opta por adaptarse puesto que tener una actitud crítica ante este contexto, no le favorece. Al principio si se me complicó un poco aplicar la teoría a la práctica, pero con el pasar del tiempo ya mejoré, y además tuve que improvisar muchas veces por la falta de insumos y esto hacía que no se aplique bien el conocimiento. (P4). En la colocación de sondas vesicales me costó aplicar la teoría a la práctica porque en la universidad hacíamos la practica en fantomas entonces no es lo mismo en un fantoma que en un paciente de verdad, ahora en el internado con la guía de la licenciada a cargo ya lo hago muy bien. (P7)

La simulación clínica en la actualidad se encuentra en boga. Limitaciones en las instituciones de salud para la práctica de estudiantes, consideraciones éticas y la tecnología de los simuladores, han posibilitado que la práctica del estudiante de carreras de la salud tenga esta opción en sus laboratorios. Sin embargo, la opinión de la estudiante anterior, da a entender que, si bien dan una idea de los procedimientos las fantomas, no lo es suficiente en la instalación de sondas en pacientes reales. Por otra parte, en un estudio realizado en Jerusalén sobre la percepción de estudiante con relación al uso de simuladores, menciona que el uso de simulaciones antes y durante la primera práctica clínica de los estudiantes de enfermería es 
una estrategia de aprendizaje útil y eficaz y que aminora los niveles de ansiedad, Khalaila, R. (2014).

\section{Sensación de incompetencia e inseguridad en la realización de procedimientos.}

La enfermería es una profesión inminentemente práctica. Con conocimiento científico y un proceso de atención, que la convierte en ciencia, pero se considera como una profesión puramente práctica. El Profesional de enfermería que se jacte debe ser ágil, rápido, eficiente, en resumen $100 \%$ destreza. Situación conocida por el estudiante quien se siente incompetente e inseguro. Hasta que me fui al internado no sabía canalizar. (P2). Me causaba preocupación por ejemplo los medicamentos, los procedimientos no porque no esté segura de mis capacidades, sino porque era la responsabilidad que tenía, yo sabía que tenía conocimientos pero no quería cometer equivocaciones. (P3).Al inicio ir con inseguridad por el miedo de que nos salga mal, esa era nuestra debilidad. (P1)

Estos pensamientos se convierten en trabas al inicio de las prácticas clínicas, se manifiestan en el lenguaje corporal del estudiante como movimientos torpes, tono de voz inaudible, lentitud en el accionar. Su aprendizaje día a día se basa en el "ensayo-error" pero lo plausible es que ese error cometido es error no repetido. Inseguridad, miedo, ansiedad son emociones comunes. Ansiedad, preocupación, duda de si mismos y miedo de hacer daño, representan dificultades no tan solo en la ejecución de procedimientos sino también en la relación terapéutica con los pacientes según Sun, F. y Moya, M. (2016). Al menos yo me di cuenta que en los tres años que estuve en la universidad nunca aprendí tanto como los tres meses de internado en el hospital Guayaquil. (P1)

Se evidencia la diferencia entre dos formas de enseñanza: el saber teórico que se encuentra en los libros y el saber practico que no está formalizado en un papel sino en las experiencias del profesional Sandoval, L. (2017), la rutina diaria del trabajo y la repitiencia de procedimientos hace pasar del sentimiento de inseguridad y sensación de incompetencia a que el estudiante gane la sensación de confianza en sí mismo y la capacidad de poder hacer todo, e inclusive más, que los licenciados experimentados. Del mismo modo como refiere Patricia Benner de principiante a principiante avanzado. Al principio si era difícil aplicar procedimientos, y cuando llegamos éramos los internos nuevos no teníamos experiencia entonces las licenciadas nos evitaban y no nos dejaban hacer nada pero después a medida que pasaban los días demostrábamos nuestra habilidad entonces ya a lo personal buscaba oportunidades para demostrarles que yo sabía y así fue. (P7)

El personal de salud evita dejar en manos inexpertas a sus pacientes, siendo negativos ante la presencia de nuevos internos, teniendo concepciones de incapacidad de los estudiantes, ante procedimientos hospitalarios, pero la seguridad en los conocimientos del estudiante y su afán de aprender más, le llevan a demostrar que si es capaz de ser digno de confianza en las 
labores hospitalarias. En las emergencias las mismas licenciadas se sabían quedar paralizadas en como pasar una sonda o canalizar rápido, en esa parte a mí me gustaba porque yo podía actuar rápido y podía ayudar en todo como succionar hacer todo ese tipo de actividades en la emergencias. (P2)

La seguridad de lo aprendido y el deseo de tener oportunidad de aprender y tener destreza permite que el estudiante se proponga demostrar no solo al personal de salud, también a ellos mismos el hacer y saber hacer.

\section{Ansiedad inicial por las relaciones interpersonales y profesionales.}

El tener buenas relaciones interpersonales con la licenciada en enfermería a cargo del área donde trabajarían, les brindaba confianza de preguntar y aprender. Sentirse respaldados, y acompañados en el proceso de aprendizaje netamente práctico fue su principal preocupación. Lo que más me preocupaba cuando entre al internado era toparme con licenciadas groseras o déspotas. (P5)

Si hubiese barreras de comunicación como la que se menciona arriba, la aplicación del conocimiento teórico a la práctica, hubiese sufrido trabas. La Licenciada en enfermería que labora en la institución es el principal facilitador de aprendizaje, es la que maneja los procedimientos administrativos, técnicos y de atención para el paciente. El interno de enfermería conoce de estos roles y competencias y desea ser participe activo de ellas, por esto, reconoce su gran preocupación de la posibilidad de no tener una buena relación con este personaje y esto, le provoca mucha ansiedad. Al principio en cómo nos íbamos a llevar con los otros compañeros que eran de otras universidades, también me preocupaba fallar en algo, en no responder o no saber, tenía miedo de preguntar porque podrían decirme que ya debía saberlo si era interna. (P3)

La enfermera del servicio simboliza la enseñanza y apoyo en los espacios clínicos. En este sentido las relaciones interpersonales entre los participantes en el aprendizaje de entorno clínico son fundamentales para el desarrollo de un ambiente de aprendizaje positivo. Durán, M. (2007). No tan solo se encuentra con la inquietud de su relación interpersonal con la enfermera a cargo sino también con sus pares de otras universidades. El hecho de quedar como incompetente ante alguna pregunta o toma de decisión causa estrés y preocupación. Hasta ahorita para que, cada licenciada gracias a Dios cada una de ellas nos han enseñado como ellas trabajan y obviamente nosotras tenemos que adaptarnos a eso. Al principio no nos dejaban solas pero ahora con el tiempo nos asignan pacientes, igual ellas nos supervisan cómo va el trabajo y todo eso. (P1)

Los enfermeros clínicos fueron percibidos como los más importantes para el desarrollo de pensamiento crítico, reflexión e intercambio de experiencias Mårtensson, G. y Uriarte, S. 
(2016). La licenciada de enfermería como facilitadora de aprendizaje es la base más fuerte que tienen los internos de enfermería en su práctica clínica. Es quien guía, enseña, y supervisa al estudiante para su fortalecimiento como profesional para la adquisición de destrezas y habilidad en el manejo del paciente enfermo.

\section{Rol profesional versus rol de ayudante del auxiliar de enfermería.}

El hecho de aun no llegar a ser profesional y ser el "interno de enfermería" crea en ellos un conflicto de rol. El estudiante conoce muy bien a dónde quiere llegar y como. Estudió día tras día en un aula de clases, adquirió bases teóricas, modelos de atención, procedimientos y demás. Conoció de sus competencias como profesional de enfermería y cuando llegó al área de trabajo a su práctica clínica pre profesional, se encuentra con que, no todo lo que le dijeron era tan cierto. O más bien, para el personal auxiliar de enfermería son un ayudante que facilitaría sus guardias. Un día no fue la auxiliar, y a mí me tocó hacerlo (asearlo al paciente), no me quejo lo hice con gusto porque son personas que necesitan de nuestra ayuda. (P7)

En las instituciones de salud se considera al estudiante, interno de Enfermería como sustituto del personal auxiliar, a pesar de ello, el afán o interés del estudiante es brindar atención holística, por esto se preocupan de las necesidades básicas del usuario, de su confort, dando atención oportuna aun en roles que no son de su competencia no obstante, el estudiante que cursa su periodo de práctica es considerado como un trabajador más de la institución cuando su principal objetivo durante este periodo corresponde al de aprendizaje y no al de realización de actividades que no van conforme a su rol. Esta situación obstaculiza el aprendizaje según Chuan, O. y Morrell, N. (2014). Lo más desagradable es cuando una auxiliar nos reportó con la jefa quejándose que nosotras las internas no la ayudábamos, cuando ella se fue a dormir un ratito, teníamos que hacer lo que le tocaba a ella, diciendo que no queríamos descartar diuresis, ni bañar pacientes cuando fue todo lo contrario. (P3)

El estudiante al tener todas estas nuevas experiencias clínicas se siente motivado y deseoso de ayudar en todo lo que pueda. En el caso anterior presentado, si bien menciona hacer las actividades del auxiliar con mucho gusto, crea en el estudiante un conflicto de rol. En la universidad se le dijo que su competencia es ser la enfermera a cargo, líder y que solo se limitaba a ciertas actividades, y que el auxiliar de enfermería estaba supeditado a sus órdenes. Pero aquí se observa que es todo lo contrario con el interno de enfermería. Se sienten imposibilitados de quejarse por el trato de las auxiliares de enfermería ya que saben que si lo hacen las relaciones interpersonales se dañarían y el aprendizaje se vería afectado. Otra experiencia desagradable fue cuando un licenciado me mando a prepararle café, mi reacción fue decirle perdóneme pero yo estoy ocupada administrando medicación y también se fue con la queja de que la interna no hace caso. (P6) 
Cuando ya recibe órdenes y especificaciones del licenciado en enfermería, el estudiante adquiere otra posición. Es el profesional al cual emula y cuando este solicita algo, siente que debe de hacerlo. Pero si la petición incluye una solicitud de algo que no tiene que ver con su aprendizaje y rol, el estudiante sin arredrase objeta y decide no hacerlo como lo manifiesta el caso anterior.

\section{Insatisfacción con la cantidad de práctica en los años universitarios.}

Los años de formación de pregrado en cada asignatura profesionalizarte, ofrecían el componente teórico y práctico. No obstante, las inferencias que hacen los estudiantes con relación a la aplicación de los conocimientos adquiridos en el día a día del quehacer enfermero evidencian la insatisfacción con ese componente práctico recibido en la universidad. Houghton, C. (2014). Está bien dar la teoría, pero debería aumentarse las horas de práctica y que se le deje al estudiante hacer las cosas, porque al principio a nosotros en el internado nos daba miedo todo nos temblaba la mano. (P6). Yo me di cuenta que en los tres años que estuve en la universidad nunca aprendí tanto como los tres meses de internado en el hospital Guayaquil. (P1)

La vida laboral sumerge al estudiante en otra etapa, ya no de aprendizaje solamente, pues ahora debe asumir otros retos, hacer y saber hacer con el conocimiento impartido en la universidad y en ocasiones poner en práctica aun lo no aprendido, lo nuevo. Pero, más bien, la adquisición de pensamiento reflexivo y crítico en situaciones adversas aminora la sensación de estrés del estudiante y lo ayuda a enfrentarlo Lee, A. (2015). El docente universitario cumple el rol importante durante la experiencia clínica del estudiante para forjar su identidad profesional Walker, S. (2014)., por lo que el abandono del preceptor, podría llevar al formando a desviar su visión inicial de lo que aprendió en la universidad. En ciertas materias debería tener más práctica, en los hospitales a veces íbamos solo dos o tres días a la semana y hacíamos todo rápido y ya pero por ejemplo creo que a los muchachos de ahora les hace falta mucho más que a nosotros. (P5)

En la totalidad de las entrevistas la petición se repetía. Mayor cantidad de prácticas durante el pregrado. Consideran que si no existiese el internado no se hubieran sentido competentes y seguros. La satisfacción percibida con sus destrezas adquiridas en el pregrado fue pobre, se relaciona con el hecho de que en las prácticas de asignatura no se verificaba si los entornos clínicos ofrecían situaciones de aprendizaje Walker, S.(2014.)

Como limitaciones principales para el aprendizaje tuvieron la injerencia de los internos de medicina que cada vez más realizan procedimientos que antes lo hacían las enfermeras. El quitar espacios les pareció como una amenaza para su campo profesional. Esos procedimientos jamás efectuados los consideran como vacíos que no se llenaran y que deberán esperar hasta cuando ya sean profesionales graduados y registrados. 


\section{Conclusiones.}

- La cantidad y calidad del componente práctico de las asignaturas de la carrera de enfermería es cuestionable. Los ambientes clínicos donde son llevados los estudiantes no son en ocasiones ricos en situaciones de aprendizaje, esto acarrea en consecuencia, estudiantes temerosos, inseguros y ansiosos cuando llega el momento de aplicar la teoría a la práctica. El estrés ocasionado por la posibilidad de fallar y hacer daño al "paciente real" no se compara a la práctica con el simulador utilizando en la práctica de laboratorio de la Universidad.

- Sin embargo, la licenciada en enfermería del área de servicio, se vuelve la aliada más importante para facilitar el aprendizaje del estudiante. Es la que guía, orienta, enseña

y fortalece la formación profesional del estudiante. Acrecentar el pensamiento crítico y capacidad resolutiva de problemas, así como forja la identidad profesional del formando.

- Enfermería ciencia y arte, profesión inminentemente práctica, pero no divorciada del conocimiento científico. Puede el estudiante ser el mejor alumno año tras año, pero será juzgado en el ámbito laboral por las habilidades prácticas. Se sugiere reforzamiento en las prácticas de asignatura, en áreas clínicas debidamente seleccionadas, donde se dé la importancia que merece a las licenciadas asistenciales como compañeras estratégicas del docente universitario. Esto sin lugar a dudas brindara al estudiante de enfermería, oportunidades y resultados de aprendizaje significativos y apropiados para su vida profesional futura.

\section{Referencias bibliográficas.}

1 Briceño VES, Ferreiro MTB, Rodríguez M del SM. La Práctica Docente Y El Desarrollo De Habilidades Intelectuales En La Formación Profesional Por Competencias. Horizontes Educacionales. 2011 Jul 16(2):43-56. Disponible en: http://www.redalyc.org/articulo.oa?id=97923680005

2. Pogre P. Enseñanza para la comprensión. Un marco para el desarrollo profesional docente. [citado 1 de septiembre de 2017]; disponible en: http://www.academia.edu/7596597/ense\%c3\%91anza_para_la_comprensi\%c3\%93n._u n_marco_para_el_desarrollo_profesional_docente 
3. Altmann TK. An evaluation of the seminal work of Patricia Benner: theory or philosophy? Contemp Nurse. 2007 Jun [cited Sep 5, 2017] ;25(1-2):114-23. doi: 10.5555/conu.2007.25.1-2.114

4. Morán L., Quezada Y., García A., González P., Godínez S., Aguilera M.. Resolver problemas y tomar decisiones, esencia de práctica reflexiva en enfermería. Análisis de la literatura. Enferm. univ [revista en la Internet]. 2016 Mar [citado 2017 Nov 25] ; 13( 1 ): 47-54. Disponible en: http://www.scielo.org.mx/scielo.php?script=sci_arttext\&pid=S166570632016000100047\&lng=es. http://dx.doi.org/10.1016/j.reu.2016.01.001.

5. Benner P. From novice to expert. Am J Nurs.1982 Mar 1;82(3):402-7. Available froom: en: https://www.ncbi.nlm.nih.gov/pubmed/6917683

6. Cárdenas Becerril L, Gómez J, Antonia M. Enseñanza y Aprendizaje del Pensamiento Reflexivo y Crítico en Estudiantes de Enfermería en Iberoameríca. 2014 Oct [citado 1 de septiembre de 2017]; Disponible en: http://ri.uaemex.mx/handle/20.500.11799/57940

7. Carrillo Algarra Ana Julia, García Serrano Lucila, Cárdenas Orjuela Claudia Marcela, Díaz Sánchez Ingrid Rocío, Yabrudy Wilches Nataly. La filosofía de Patricia Benner y la práctica clínica. Enferm. glob. [Internet]. 2013 Oct [citado 2017 Nov 07] ; 12( 32 ): 346-361. Disponible en:

http://scielo.isciii.es/scielo.php?script=sci_arttext\&pid=S1695$61412013000400021 \& \operatorname{lng}=$ es.

8. Onwuegbuzie AJ, Dickinson WB, Leech NL, Zoran AG. Un marco cualitativo para la recolección y análisis de datos en la investigación basada en grupos focales. Paradigmas. 2013 Jul 22;3(2):127-57. Disponible en: https://dialnet.unirioja.es/servlet/articulo?codigo $=3798215$

9. Cadenas DMR. El Rigor en la Investigación Cualitativa: Técnicas de Análisis, Credibilidad, Transferibilidad y Confirmabilidad. SINOPSIS EDUCATIVA Revista venezolana de investigación. 2016 May 18;7(1):17-26. Disponible en: http://revistas.upel.edu.ve/index.php/sinopsis_educativa/article/view/3539/1715

10. Carrera H, M R. La investigación cualitativa a través de entrevistas: su análisis mediante la teoría fundamentada. [Internet]. 2014 [citado 25 de noviembre de 2017]; Disponible en: https://idus.us.es/xmlui/handle/11441/36261

11. Martínez Rodríguez L, Venceslao Pueyo M, Ramió i Jofre A. Crisis de cuidados: 
Percepciones del cuidado en los estudiantes de enfermería. Psicoperspectivas. noviembre de 2016;15(3):135-45. doi: 10.5027/psicoperspectivas-Vol15-Issue3fulltext-777

12. Khalaila R. Simulation in nursing education: An evaluation of students' outcomes at their first clinical practice combined with simulations. Nurse Education Today. 2014 Feb 1;34(2):252-8. doi: 10.1016/j.nedt.2013.08.015

13. Sun F-K, Long A, Tseng YS, Huang H-M, You J-H, Chiang C-Y. Undergraduate student nurses' lived experiences of anxiety during their first clinical practicum: A phenomenological study. Nurse Education Today. 2016 Feb 1;37:21-6. doi: 10.1016/j.nedt.2015.11.001.

14. Moya Nicolás María, Larrosa Sánchez Sandra, López Marín Cristina, López Rodríguez Irene, Morales Ruiz Lidia, Simón Gómez Ángela. Percepción del estrés en los estudiantes de enfermería ante sus prácticas clínicas. Enferm. glob. [Internet]. 2013 Jul [citado 2017 Nov 07] ; 12( 31 ): 232-253. Disponible en: http://scielo.isciii.es/scielo.php?script=sci_arttext\&pid=S1695$61412013000300014 \& \operatorname{lng}=$ es.

15. Sandoval LEP, Prado ALR. Relación entre teoría y praxis en la formación de profesionales de enfermería: revisión integradora de literatura. Enfermería Global. 28 de junio de 2017;16(3):679-706. Disponible en: http://revistas.um.es/eglobal/article/view/269261

16. Durán MJP, Rodríguez RA. Situaciones de las prácticas clínicas que provocan estres en los estudiantes de enfermería. Enfermería global: Revista electrónica semestral de enfermería. Mayo 2007;6(1):1-12. Disponible en: http://revistas.um.es/eglobal/article/view/264

17. Mårtensson G, Löfmark A, Mamhidir A-G, Skytt B. Preceptors' reflections on their educational role before and after a preceptor preparation course: A prospective qualitative study. Nurse Education in Practice. 2016 Jul 1;19:1-6. doi: 10.1016/j.nepr.2016.03.011.

18. Uriarte S, Ponce G, Bernal M. Vivencias cotidianas en espacios clínicos del estudiante de enfermería. Enfermería Universitaria. 1 de julio de 2016;13(3):171-7. Disponible en: http://www.redalyc.org/articulo.oa?id=358746914006

19 Chuan OL, Barnett T. Student, tutor and staff nurse perceptions of the clinical learning environment. Nurse Educ Pract. 2012 Jul 1;12(4):192-7. doi: 
10.1016/j.nepr.2012.01.003.

20. Morrell N, Ridgway V. Are we preparing student nurses for final practice placement? British Journal of Nursing. 2014 May 21;23(10):518-23. doi:

10.12968/bjon.2014.23.10.518

21. Houghton CE, Casey D, Shaw D, Murphy K. Students' experiences of implementing clinical skills in the real world of practice. J Clin Nurs. 2013 Jul 1;22(13-14):1961-9. doi: 10.1111/jocn.12014.

22. Lee AK, You HS, Park IH. Affecting Factors on Stress of Clinical Practice in Nursing Students. Journal of Korean Academy of Nursing Administration. 2015 Mar 1;21(2):154-63. Available from: https://doi.org/10.11111/jkana.2015.21.2.154

23. Walker S, Dwyer T, Broadbent M, Moxham L, Sander T, Edwards K. Constructing a nursing identity within the clinical environment: The student nurse experience. Contemporary Nurse. 2014 Dec 1;49(1):103-12. doi: 10.5172/conu.2014.49.103.

24. Bisholt B, Ohlsson U, Engström AK, Johansson AS, Gustafsson M. Nursing students' assessment of the learning environment in different clinical settings. Nurse Education in Practice. 2014 May 1;14(3):304-10. doi: 10.1016/j.nepr.2013.11.005.

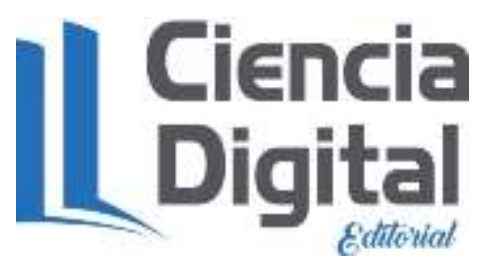




\section{Para citar el artículo indexado.}

Franco J., Oviedo R., Piedra K., \& Torres A. (2018). La formación práctica en enfermería un estudio cualitativo en los internos que cursan sus prácticas clínicas. Revista electrónica Ciencia Digital 2(1), 46 -59. Recuperado desde:

http://www.cienciadigital.org/revistascienciadigital/index.php/CienciaDigital/article/view/4 $/ 4$

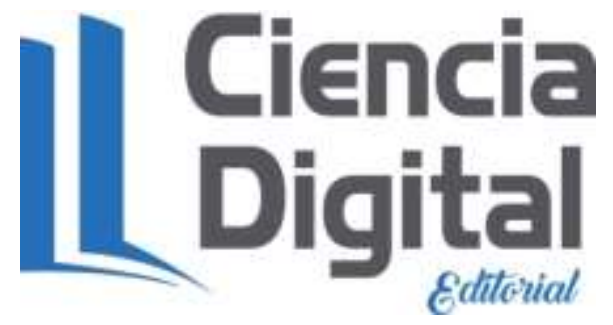

El artículo que se publica es de exclusiva responsabilidad de los autores y no necesariamente reflejan el pensamiento de la Revista Ciencia Digital.

El articulo queda en propiedad de la revista y, por tanto, su publicación parcial y/o total en otro medio tiene que ser autorizado por el director de la Revista Ciencia Digital.
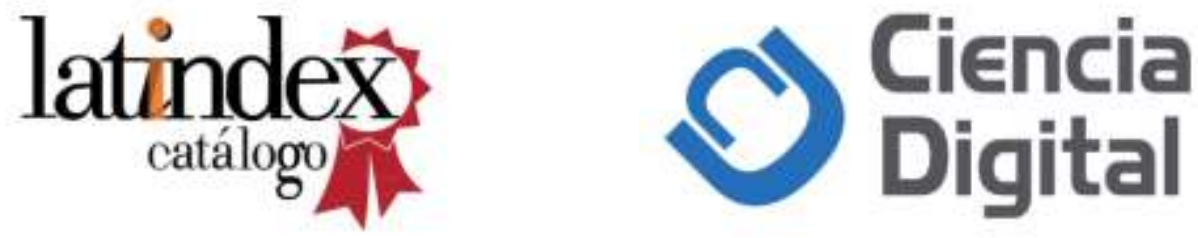